\title{
EFECTOS DEL PROGRAMA DE RECUPERACIÓN PSICOPEDAGÓGICA EN ALUMNOS CON DIFICULTADES DE APRENDIZAJE DE LAS INSTITUCIONES EDUCATIVAS DE PRIMARIA, HUÁNUCO - 2011
}

\author{
EFFECTS OF RECOVERY PROGRAM PSYCHOPEDAGOGICAL IN STUDENTS \\ WITH LEARNING DIFFICULTIES OF ELEMENTARY SCHOOLS, HUÁNUCO - 2011
}

\author{
Lilia Campos C. y Miguel Jaimes C. * \\ Universidad Nacional Hermilio Valdizán, Huánuco, Perú
}

(Recibido 13/02/2013, Aceptado 14/05/2013)

\begin{abstract}
RESUMEN
El estudio tiene como objetivo determinar los efectos de un programa integral de recuperación psicopedagógica en los alumnos con dificultades de aprendizaje de las Instituciones de Educación Primaria de la ciudad de Huánuco.

Se ha tenido como muestra a 61 alumnos de Primero a Sexto Grado a quienes se les ha aplicado programas de acuerdo a las dificultades detectadas, se trabajó con los Docentes, quienes aplicaban los programas en el aula y los padres fueron capacitados para el acompañamiento escolar.

El diseño es cuasiexperimental, con pre y postprueba en un solo grupo, utilizando los instrumentos psicológicos del EVALÚA, la prueba de Percepción Visomotriz de Bender, y Factor "G" de Cattell. Para la evaluación a los Docentes se elaboraron Listas de Cotejo y entrevistas a los padres de familia; luego se procesaron los datos donde los resultados determinaron la eficacia del programa. Así mismo se encontraron diferencias significativas en los resultados entre el pre y el post-test, incrementándose el porcentaje de alumnos que superaron sus dificultades.
\end{abstract}

Palabras clave: dificultades de aprendizaje, programa de recuperación psicopedagógica

\begin{abstract}
The study has been aimed at determining the effects of a recovery program in educational psychology students with learning difficulties of Primary Education Institutions Huanuco for which as shown has 61 students with learning difficulties Educational Institutions Primary Huanuco. Was taken as a sample to 61 students in first through sixth grade who have been applied programs according to the identified challenges, we worked with teachers who implemented the programs in the classroom and parents who were trained to accompany school their children.
\end{abstract}


EFECTOS del PROGRAMA DE RECUPERACIÓN PSICOPEDAGÓGICA EN ALUMNOS CON DIFICULTADES DE APRENDIZAJE...

The quasi-experimental design with pre and post test in a single group. Using psychological instruments tests such as the tester, test Bender visual-motor perception, and Cattell. For the evaluation to be developed Teachers' checklists and observation and interviews with parents. And then processed the data where the results showed the effectiveness of the program. Also significant differences in outcomes between the pre and post test, increasing the percentage of students who overcame their difficulties.

Keywords: learning disabilities, psychology recovery program

\section{INTRODUCCIÓN}

En la actualidad se han llevado a cabo pocos estudios en el campo de la Educación que, en forma sistemática trabajen directamente con los alumnos, docentes, y padres de familia, utilizando el programa como la estrategia de trabajo que permita resolver y superar las dificultades de aprendizaje que presentan los alumnos de Educación Primaria.

Con alguna frecuencia, los maestros tienen alumnos con diversos grados de dificultad para aprender, es decir alumnos con limitaciones para memorizar la información social que se les proporciona en cada lección y que, como consecuencia de esas dificultades, no logran desarrollarse de modo integral. Dificultades de aprendizaje es un término general que describe problemas del aprendizaje específicos, y puede causar que una persona tenga dificultades aprendiendo y usando ciertas destrezas, como en la lectura, ortografía, escuchar, hablar, razonamiento matemático, comprensión y escritura.

Pedro Ortíz (1998) considera las dificultades de aprendizaje como defectos en la estructuración de la conciencia, que pueden afectar la formación de la personalidad y que pueden ser causados por un defecto del desarrollo del sistema del habla o de los componentes de la conciencia en sí, por fallas en el desarrollo del cerebro, de extensión tanto global como parcial, y que ocurren tanto en la epigénesis como en la sociocínesis de la personalidad.

Además, es probable que la mayor incidencia de estos problemas sea resultado de las condiciones en que se gestan, nacen y empiezan a crecer y formarse las personas, pues algún resultado tiene que generar la situación de madres jóvenes y solteras, madres que no tienen control en su gestación, que tienen mayor probabilidad de alto riesgo, infecciones, así como las condiciones en que se educan y forman los niños y niñas en medio de la pobreza y la informalidad, la desnutrición, estilos de vida desfavorables en los hogares, es de esperar que defectos tan mínimos, como un simple retardo de adquisición del habla o de la lectura, tengan repercusiones no solo en el aprendizaje sino más profundas y duraderas en la personalidad que se forma en estas condiciones.

Según el Índice Global de Escolaridad (Ministerio de Educación, 2008), solo el $29 \%$ de alumnos culminan la Primaria a la edad esperada de 12 años y el resto culmina después, esto hace suponer que la mayoría ha tenido retraso escolar. Así mismo, se observa en la Región Huánuco una tasa de conclusión de educación 
primaria de $78,8 \%$ en una edad de 15 a 17 años; y solo el $54,1 \%$ de estudiantes concluyen su primaria en las edades de 12 a 14 años, el perfil educativo logrado por medio de las coberturas de sistema, es de 88,2\% en el grupo de 6 a 11 años de edad en comparación a nivel nacional de $94,7 \%$. Por otro lado, se evidencia una frecuencia de 151,390 estudiantes que estudian el nivel primario y el grupo etáreo con mayor frecuencia que no van a la escuela recae en los 4 a 5 años de edad (nivel inicial). Esta problemática se refleja en la educación primaria porque los alumnos que inician su etapa de escolaridad no han realizado el aprestamiento y tardíamente se les prepara en habilidades cognitivas, motrices, espaciales que de acuerdo a las teorías psiconeurológicas también afectan al desarrollo normal en los niños que influyen en su desempeño escolar más adelante.

Hugo Díaz (2007) en su análisis sobre el Panorama Actual de la educación señala que, la característica principal del comportamiento de la matrícula al 2011 es continuar la reducción de la educación primaria -en más de un cuarto de millón de alumnos- y que la educación secundaria siga creciendo. Para ello se necesitan amplias acciones preventivas para evitar la repetición de grado o el abandono estudiantil en las escuelas. Si el Estado invirtiese el 10\% de los 538 millones de soles que cuesta anualmente la repetición y el abandono escolar en programas de ampliación de la jornada laboral para los alumnos en riesgo de repetir el año escolar, seguramente el costo de los fracasos escolares podría disminuir en un 50 a $60 \%$.

En un trabajo de investigación realizado en la Universidad Nacional Hermilio Valdizán de Huánuco (Ramírez, R. Campos L. y otros 2008) sobre el diagnóstico de alumnos con problemas de aprendizaje en las Instituciones Educativas de zonas marginales de Huánuco, con alumnos del Primero al Sexto Grado de Primaria de los distritos de Huánuco y Amarilis, se ha encontrado que el $81 \%$ de alumnos con dificultades de aprendizaje presentan niveles por debajo del promedio en cuanto a comprensión lectora, el 26\% presentan nivel Normal Bajo, el 51\% nivel Inferior y el $4 \%$ nivel deficiente. En Razonamiento Lógico el $71 \%$ de alumnos se encuentran por debajo del promedio, el $33 \%$ se ubican en niveles Normal Bajo, el $26 \%$ nivel Inferior y un $12 \%$ con nivel Deficiente.

Son múltiples las variables asociadas a estas dificultades, siendo las más relevantes la situación de pobreza de las familias, el problema de la desnutrición que en la actualidad se registra a nivel de Huánuco el $49 \%$, la no existencia de programas de intervención ni Docentes especializados en el tratamiento de alumnos con dificultades específicas de aprendizaje; puesto que los Programas de Capacitación del Ministerio de Educación están orientados al manejo operativo de los planes curriculares centrados en la enseñanza y no en el aprendizaje de los niños; no toman en cuenta las diferencias socioculturales en las que se aplican, menos aún las diferencias individuales de los alumnos.

Es a partir de esta problemática la necesidad de brindar una atención oportuna y especializada a los alumnos de Educación Primaria por las dificultades que presentan a nivel perceptivo, psicomotriz, de aprestamiento, comprensión lectora y razonamiento lógico entre otros. 
EFECTOS DEL PROGRAMA DE RECUPERACIÓN PSICOPEDAGÓGICA EN ALUMNOS CON DIFICULTADES DE APRENDIZAJE...

Como consecuencia de lo expuesto, los problemas de la presente investigación se plantean de la siguiente manera:

¿En qué medida un programa de recuperación psicopedagógica influye en la mejora del rendimiento escolar de los alumnos con dificultades de aprendizaje de las Instituciones Educativas de Primaria de Huánuco?

¿Cuáles son las dificultades de aprendizaje más frecuentes que presentan los alumnos de Educación Primaria en el aula?

\section{OBJETIVOS}

- Determinar los efectos de un programa de recuperación psicopedagógica en alumnos con dificultades de aprendizaje de las Instituciones de educación primaria de Huánuco.

- Identificar las dificultades de aprendizaje más frecuentes en los alumnos de educación Primaria de Huánuco.

- Evaluar y comparar los niveles de desempeño en el manejo de estrategias psicopedagógicas de los Docentes para apoyar a los alumnos con dificultades de aprendizaje.

\section{HIPÓTESIS}

$\mathrm{H}_{1}$ : La aplicación de un programa de recuperación psicopedagógica influye en la mejora del rendimiento escolar de los alumnos con dificultades de aprendizaje de las Instituciones de Educación Primaria de Huánuco.

$\mathrm{H}_{10}$ : La aplicación de un programa integral con los alumnos, docentes y la familia no influye en la mejora del rendimiento escolar de los alumnos con dificultades de aprendizaje de las Instituciones de Educación Primaria de Huánuco.

$\mathrm{H}_{2}$ : La asesoría en el manejo de estrategias a los Docentes para la intervención de los alumnos con dificultades de aprendizaje influye en el incremento de su rendimiento escolar.

$\mathrm{H}_{20}$ : La asesoría en el manejo de estrategias a los Docentes para la intervención de los alumnos con dificultades de aprendizaje no influye en el incremento de su rendimiento escolar

\section{MÉTODO}

El diseño cuasiexperimental, con pre y postprueba, en un solo grupo, pues se trata de estudiar el efecto de un programa de recuperación psicopedagógica para mejorar el rendimiento escolar de los alumnos de Educación primaria, teniendo en cuenta el contexto familiar y la intervención pedagógica de los Docentes (Hernández, Fernández y Baptista 2006). 
La muestra estuvo conformado por 61 estudiantes con dificultades de aprendizaje de tres Instituciones Educativas Estatales de Primaria a quienes se les aplicó los instrumentos psicológicos del EVALÚA, Prueba de Percepción Visomotriz de Bender y la prueba del Factor "G" de Cattel para el diagnóstico y la aplicación de los programas de acuerdo a sus dificultades; 28 docentes quienes realizaron el reforzamiento en sus aulas de acuerdo a programas específicos y los padres de familia que fueron sensibilizados para el acompañamiento escolar a sus hijos y capacitados en nutrición y estilos de vida saludables.

\section{RESULTADOS}

En la Tabla N. ${ }^{\circ} 1$ se presentan los resultados de la evaluación de entrada a la muestra y la evaluación luego de aplicado el programa de recuperación psicopedagógica, observando el incremento en los indicadores de aprendizaje siendo mayor en noción numérica en un 36\%, coordinación motora fona en un $25 \%$ y escritura en un $23 \%$ y menor en un $10 \%$ en cálculo. En promedio se ha incrementado superar las dificultades de aprendizaje de los alumnos de la muestra en un $20 \%$. Y que de un $27 \%$ de alumnos que no mostraban dificultades se incrementó luego de aplicado el programa a un $46 \%$ de la muestra, por lo que se demostró la efectividad del programa.

Así mismo podemos señalar que estadísticamente también se demostró diferencias significativas entre la evaluación del pretest y la evaluación del posttest luego de aplicado el programa, mediante la técnica del " $t$ " de Student.

Tabla N. ${ }^{\circ}$ 1. Resultados de dificultades de aprendizaje de los alumnos de las I.E. de Primaria de Huánuco-2011

\begin{tabular}{lccc}
\hline \multicolumn{1}{c}{ Dificultades de aprendizaje } & Inicio & Término & Incremento \\
\hline - Dificultades de atención & $17 \%$ & $33 \%$ & $16 \%$ \\
- Dificultades de retención & $35 \%$ & $52 \%$ & $17 \%$ \\
- Comprensión lectora & $30 \%$ & $50 \%$ & $20 \%$ \\
- Dificultades en noción numérica & $22 \%$ & $58 \%$ & $36 \%$ \\
- Dificultades en cálculo & $15 \%$ & $25 \%$ & $10 \%$ \\
- Dificultades de lectoescritura & $10 \%$ & $26 \%$ & $16 \%$ \\
- Dificultades en coordinación motora fina & $40 \%$ & $63 \%$ & $23 \%$ \\
- Dificultades en conciencia fonológica & $20 \%$ & $45 \%$ & $25 \%$ \\
- Dificultades de discriminación visual & $50 \%$ & $65 \%$ & $15 \%$ \\
\hline
\end{tabular}


EFECTOS DEL PROGRAMA DE RECUPERACIÓN PSICOPEDAGÓGICA EN ALUMNOS CON DIFICULTADES DE APRENDIZAJE...



Figura N. ${ }^{\circ}$ 1. Resultados del Pre y Post Test del Programa de Recuperación psicopedagógica en alumnos con dificultades de aprendizaje de las I.E. de Primaria de Huánuco-2011.

En la Tabla N. ${ }^{\circ} 2$, en los factores determinantes de las dificultades de aprendizaje de los alumnos según los padres de familia, el $33 \%$ de padres reconocen su falta de apoyo en las tareas escolares y problemas personales de los alumnos en un $31 \%$, sin embargo un $27 \%$ de padres señalan que es por falta de exigencia de los docentes.

Tabla $\mathbf{N}^{\circ}$ 2. Factores determinantes de las dificultades aprendizaje de los alumnos, según los padres de familia

\begin{tabular}{lc}
\hline \multicolumn{1}{c}{ Factores } & Porcentaje \\
\hline - Problemas personales de los alumnos & $31 \%$ \\
- Falta de exigencia en su Institución & $27 \%$ \\
- Educativa & \\
- Por enfermedades que adolecen & $09 \%$ \\
- Falta de apoyo de los padres & $33 \%$ \\
\hline
\end{tabular}

En la Tabla N. ${ }^{\circ} 3$, el $43 \%$ de docentes refieren que los alumnos con dificultades de aprendizaje no tienen apoyo de sus padres en sus hogares, y un $13 \%$ por aspectos metodológicos. 
Tabla N. ${ }^{\circ}$ 3. Factores determinantes de las dificultades aprendizaje de los alumnos, según los padres de familia

\begin{tabular}{lc}
\hline \multicolumn{1}{c}{ Factores } & Porcentaje \\
\hline - Problemas personales de los alumnos & $28 \%$ \\
- Falta de acompañamiento en las tareas por sus padres & $43 \%$ \\
- Por antecedentes pedagógicos inadecuados & $16 \%$ \\
- Aspectos metodológicos inadecuados & $13 \%$ \\
\hline
\end{tabular}

\section{DISCUSIÓN}

A partir de los datos procesados y teniendo en cuenta los objetivos e hipótesis planteados podemos señalar lo siguiente:

De acuerdo a la hipótesis general, la aplicación del programa de recuperación psicopedagógica ha sido efectiva se encontró diferencias estadísticamente significativas entre las evaluaciones antes y después de la aplicación del programa de recuperación psicopedagógica.

Esto resultados nos demuestran que una enseñanza individualizada y teniendo en cuenta las dificultades específicas que presentan los alumnos les permite superar y nivelarse de acuerdo a las exigencias educativas porque son diversas las causas que influyen. Así, Coles (1987) señala que las dificultades de aprendizaje surgen en el contexto de la compleja red de interacciones sociales en las que se desarrolla el alumno, donde no solo va construyendo una serie de conocimientos sino que también se van conformando las actitudes, valores y motivación necesarias para tener éxito en los aprendizajes escolares. Estas interacciones se producen en el medio familiar y escolar y, en ocasiones, no son las adecuadas para responder a las exigencias escolares; esto en razón que en la escuela no se diferencian las dificultades específicas con las dificultades generales y los Docentes señalan que la gran mayoría de estos alumnos no tienen el apoyo de sus padres señalando que son la causa principal de las dificultades de aprendizaje de sus alumnos.

Según la hipótesis señalada sobre las características de los alumnos de la muestra en mayor porcentaje (49\%) se encuentran por debajo del nivel promedio y solo un $(15 \%)$ se ubican en el nivel superior y muy superior.

Sin embargo debemos señalar que se debe tener en cuenta el criterio de discrepancia entre rendimiento y capacidad intelectual (CI), tal como señala (Rodrigo y Jiménez, 2000) que en algunas investigaciones que han realizado aportan evidencia de que el CI no explica diferencias entre buenos y malos lectores, así como otros estudios con disléxicos.

Teniendo en cuenta los resultados de la prueba de Habilidades de Aprendizaje un $(58 \%)$ de alumnos presentan habilidades de aprendizaje por debajo del nivel promedio frente a un (29\%) que se ubican por encima del promedio. 
En relación a problemas de aprendizaje encontramos que el $71 \%$ de alumnos se encuentran por debajo del nivel promedio. Estos datos no hacen más que confirmar los bajos rendimientos académicos de los escolares, puestos al descubierto a través de los estudios realizados por UNESCO y el consorcio PISA (PREAL/GRADE, 2003). Además es la evidencia de la crisis educativa cuya responsabilidad debe ser compartida por los diferentes sectores de la sociedad.

Finalmente, sobre la influencia de los padres en el aprendizaje de sus hijos relacionado con la falta de acompañamiento escolar que ha sido reconocido por los mismos padres, influye en las dificultades del aprendizaje de sus hijos. Así mismo debemos señalar no solo son los factores pedagógicos y sociofamiliares que influyen en las dificultades de aprendizaje de los alumnos, sino también tienen que ver con los ritmos y estilos de aprendizaje de los alumnos y en algunos casos aspectos maduracionales y neuropsicológicos.

Keefe (1988) plantea que una construcción particular permite definir los estilos de aprendizaje, o sea aquellos rasgos cognitivos, afectivos y fisiológicos que sirven como indicadores relativamente estables, de cómo los alumnos perciben interacciones y responden a sus ambientes de aprendizaje". En tal sentido se proponen tratamientos psicoeducacionales integrales con pretensiones de una fuerte validez extrema y ecológica, es decir, procurando adaptarse lo más posible a las situaciones reales en las que el niño se desenvuelve. Así el Modelo de estrategias de intervención de Schumaher, Deshler y Ellis (1986) que considera componentes del curriculum, instruccionales y organizacionales para los programas de tratamiento de los alumnos con dificultades de aprendizaje.

\section{CONCLUSIONES}

Existen diferencias porcentuales y estadísticas entre las evaluaciones del pretest y posttest, por lo que se afirma la efectividad del programa de recuperación psicopedagógica en alumnos con dificultades de aprendizaje.

Los alumnos con dificultades de aprendizaje que han conformado la muestra de estudio, presentan en mayor porcentaje niveles intelectuales, visomotrices y de habilidades de aprendizaje por debajo del nivel promedio.

Los docentes señalaron que los alumnos con dificultades de aprendizaje requieren de atención especializada en muchos casos y que sumado a ello los problemas personales y la falta de acompañamiento de sus padres influyen para que no superen sus dificultades de aprendizaje http://open.spotify.com/ track/7KJED0HLG7bciJIynGX13h.

Se ha determinado que entre otros factores, los socioculturales influyen en el rendimiento escolar de los alumnos como son el problema de la desnutrición, las condiciones de pobreza no solo económicas sino educativas y culturales de las familias, por lo que los programas tienen que ser integrales. 
Finalmente debemos señalar que las dificultades generales, debido a factores educacionales como falta de aprestamiento, aspectos socioculturales, problemas emocionales pueden ser superados con programas psicopedagógicos, sin embargo las dificultades específicas deben ser tratados de acuerdo a su naturaleza de manera especializada.

\section{REFERENCIAS BIBLIOGRÁFICAS}

Aguirre, R. (2000). Dificultades de Aprendizaje de la Lectura y la Escritura. Revista Educere pp. 140-167: Universidad de los Andes, Mérida-Venezuela.

Baddely, A.D. (1999). "Working memory". En: García M., J. et al. Comprensión lectora y memoria operativa. Barcelona: Edit. Paidós.

Bravo, L. (1991). Psicología de las dificultades de Aprendizaje Escolar. Ed. Universitaria: Santiago de Chile. Calderón, M. (2000). Trastorno por déficit de atención: Evaluación, diagnóstico y tratamiento. Tesis para optar título profesional de la Universidad Nacional Mayor de San Marcos.

Cuetos, F. (1996). Psicología de la lectura. Barcelona: Praxis

Claux, M. L. y La Rosa (2004). La Comprensión de Lectura en el aula: una experiencia significativa. Lima: Pontificia Universidad Católica.

Coles (1987). Mental Health of Children and Adolescents in Great Britain. London: Stationery Office.

Díaz Hugo (2007). Evaluación censal a estudiantes. Perú.

Keefe James (1988). Aprendiendo Perfiles de Aprendizaje: manual de examinador, Reston, VA: Asociación Nacional de Principal de Escuela de Secundaria.Buenos Aires.

Mares, F. (1995). Efectos de un programa de estimulación sobre habilidades básicas para el aprendizaje de la lecto-escritura en una muestra urbano marginal de la ciudad de Iquitos. Tesis para optar título de la Universidad Nacional Mayor de San Marcos.

Fernández, F. (1995). La percepción del profesor como indicador de dificultades de aprendizaje. Revista Psicothema. Universidad de Oviedo, Año/Vol. 7 N. ${ }^{\circ}$ 2, pp. 309-316, España.

Guzmán, R.; Jiménez, J. et cols. (2004). Evaluación de la velocidad de nombrar en las dificultades de aprendizaje de la lectura. Revista Psicothema Vol. 16, N. ${ }^{\circ} 3$, pp. 442-447. Universidad de Oviedo, España.

Ramírez, R, Campos, L. (2008). Variables asociadas a las dificultades de aprendizaje en estudiantes de zonas marginales de Huánuco. UNHEVAL. Huánuco

Schumaher, Deshler y Ellis(1986). Estrategias de Ayuda para niños con problemas de memoria, atención y lenguaje. Universidad de Kansas. EE.UU.

Ortíz, Pedro (1998). La Formación de la Personalidad. Lima: Dimaso Editores. 